\title{
Los niveles de acetilcolinesterasa en pacientes con inestabilidad hemodinámica descartan sepsis como causa de choque
}

\author{
Enrique Monares Zepeda, ${ }^{\star}$ Edgar Andrés Ojeda Izquierdo, ${ }^{\ddagger}$ Alberto Zamora Palma, \\ Carlos Galindo Martín, * Julia Cumandá Cuesta Torres*
}

\section{RESUMEN}

Introducción: La detección temprana de sepsis es fundamental para disminuir la mortalidad de este padecimiento. Hasta el momento, ninguna escala clínica ni biomarcador ha sido útil en su diagnóstico y detección precoz. Los niveles de actividad de la acetilcolinesterasa se han propuesto como un biomarcador muy prometedor para la detección de sepsis y choque séptico.

Objetivo: Estudiar la utilidad de los niveles de acetilcolinesterasa en el diagnóstico de sepsis.

Material y métodos: Estudio observacional y prospectivo, en pacientes de la Unidad de Cuidados Intensivos del Hospital San Ángel Inn Universidad con diagnóstico de inestabilidad hemodinámica, clasificados con presencia o no de sepsis, tomándose niveles de acetilcolinesterasa al ingreso.

Resultados: Se evaluaron 44 pacientes, $27 \%$ con sepsis. Los valores de acetilcolinesterasa tuvieron validez diagnóstica para predecir sepsis (AUC: 0.852 IC 95\%: 0.726-0.977, p < 0.001), con un punto de corte de 3,956 U/L, sensibilidad $=81 \%$, especificidad $=75 \%$. El valor de neutrófilos con corte $<74 \%$ también tuvo valor predictor de sepsis (AUC: 0,710 IC 95\%: 0,512-0,907, $p=$ 0.034 ) sensibilidad $=75 \%$, especificidad $=75 \%$.

Conclusiones: En pacientes con inestabilidad hemodinámica con niveles de acetilcolinesterasa mayores de $4,000 \mathrm{U} / \mathrm{L}$ se descarta sepsis como causa de choque.

Palabras clave: Actividad de acetilcolinesterasa sérica, biomarcador, sepsis, choque séptico, SIRS.

\section{SUMMARY}

Introduction: Early detection of sepsis is essential to reduce mortality from this condition. So far, no clinical scale or biomarker has been useful in its diagnosis and early detection. Levels of acetylcholinesterase activity have been proposed as a very promising biomarker for the detection of sepsis and septic shock.

Objective: To study the usefulness of acetylcholinesterase levels in the diagnosis of sepsis.

Material and methods: Observational and prospective study in patients of the Intensive Care Unit of the Hospital San Ángel Inn Universidad with diagnosis of hemodynamic instability, classified with or without sepsis, taking acetylcholinesterase levels at admission.

Results: 44 patients were evaluated, $27 \%$ with sepsis. The acetylcholinesterase values presented a normal distribution and had diagnostic validity to predic sepsis (AUC: 0.852 95\% Cl: 0.726-0.977, $p<0.001$ ), with a cut-off value of < $3,956 \mathrm{U} / \mathrm{L}$, sensitivity $=81 \%$, specificity $=75 \%$. The value of neutrophils with a cut $<74 \%$ also had a predictive value of sepsis (AUC: $0.71095 \%$ Cl: 0.512 $0.907, p=0.034$ ) sensitivity $=75 \%$, specificity $=75 \%$

Conclusions: In patients with hemodynamic instability with acetylcholinesterase levels greater than 4,000 U/L, sepsis is ruled out as the cause of shock.

Key words: Serum acetylcholinesterase activity, biomarker, sepsis, septic shock, SIRS.

\section{RESUMO}

Introdução: A detecção precoce da sepse é fundamental para reduzir a mortalidade desta doença. Até agora nenhuma escala clínica ou biomarcador tem sido útil em seu diagnóstico e detecção precoce. Os níveis de atividade da acetilcolinesterase têm sido propostos como um biomarcador muito promissor para a detecção de sepse e choque séptico.

Objetivo: Estudar a utilidade dos níveis de acetilcolinesterase no diagnóstico da sepse.

Material e métodos: Estudo observacional e prospectivo, realizado em pacientes da unidade de terapia intensiva do Hospital Universitário San Ángel Inn, com diagnóstico de instabilidade hemodinâmica, classificados com ou sem a presença de sepse, obtendo os níveis de acetilcolinesterase na admissão.

\footnotetext{
* Hospital San Ángel Inn Universidad.

₹ Hospital General de México «Dr. Eduardo Liceaga».

Recepción: 01/09/2017. Aceptación: 06/09/2018.

Este artículo puede ser consultado en versión completa en http://www.medigraphic.com/medicinacritica
}

Resultados: Foram avaliados 44 pacientes, 27\% com sepse. Os valores de acetilcolinesterase apresentaram validade diagnóstica para predizer sepse (AUC: 0.852 IC 95\%: 0.726-0.977, $p<0.001$ ), com valor de corte $3956 \mathrm{U} / \mathrm{L}$, sensibilidade $=81 \%$, especificidade $=75 \%$. O valor de neutrófilos com um corte $<74 \%$ também teve um valor preditivo de sepsis (AUC: 0.710 IC 95\%: 0.512$0.907, p=0.034$ ) sensibilidade $=75 \%$, especificidade $=75 \%$.

Conclusões: Em pacientes com instabilidade hemodinâmica com níveis de acetilcolinesterase superiores a 4,000 U/L a sepse é descartada como causa de choque.

Palavras-chave: Atividade da acetilcolinesterase sérica, biomarcador, sepse, choque séptico, SIRS.

\section{INTRODUCCIÓN}

Todavía no se ha encontrado un biomarcador ideal, con alta sensibilidad, especificidad y rentabilidad. ${ }^{1}$ Los niveles de acetilcolinesterasa son muy prometedores para el diagnóstico de sepsis, especialmente para diferenciar otras causas de inestabilidad hemodinámica con datos de respuesta inflamatoria sistémica, ya que la conducción colinérgica neural desempeña un papel importante en la regulación de respuesta inmune durante la inflamación. ${ }^{2}$ La vía colinérgica antiinflamatoria se encuentra compuesta por el nervio vago y su principal neurotransmisor, acetilcolina. Existe comunicación entre el nervio vago (componente aferente) y el cerebro, compartiendo información acerca de los procesos inflamatorios que ocurren en la periferia. ${ }^{3}$ El aumento de la estimulación vagal durante la inflamación inhibe la liberación periférica de citosinas; específicamente, la disminución del TNF sistémico a través de una vía que requiere de la subunidad $\alpha 7$ del receptor nicotínico de acetilcolina (Figura 1). ${ }^{4}$

Existen dos tipos de acetilcolinesterasa. La verdadera acetilcolinesterasa o colinesterasa I es encontrada en los eritrocitos, pulmones, bazo, terminaciones nerviosas y cerebro. Hidroliza el neurotransmisor acetilcolina en la sinapsis y facilita la despolarización nerviosa. La pseudocolinesterasa o colinesterasa II se encuentra en hígado, páncreas, sustancia blanca cerebral y plasma. Es usada como marcador de función hepática y su rol biológico aún no está totalmente dilucidado.

La fisiopatología de la disminución de la acetilcolinesterasa en pacientes sépticos aún no está totalmente entendida; algunas hipótesis postulan la progresión de la enfermedad, toxinas bacterianas que desencadenan la liberación de neutrófilos, aumento de la permeabilidad capilar responsable de la pérdida de colinesterasa transcapilar, aumento en el catabolismo y la inhibición de acetilcolinesterasa por citosinas inflamatorias. 


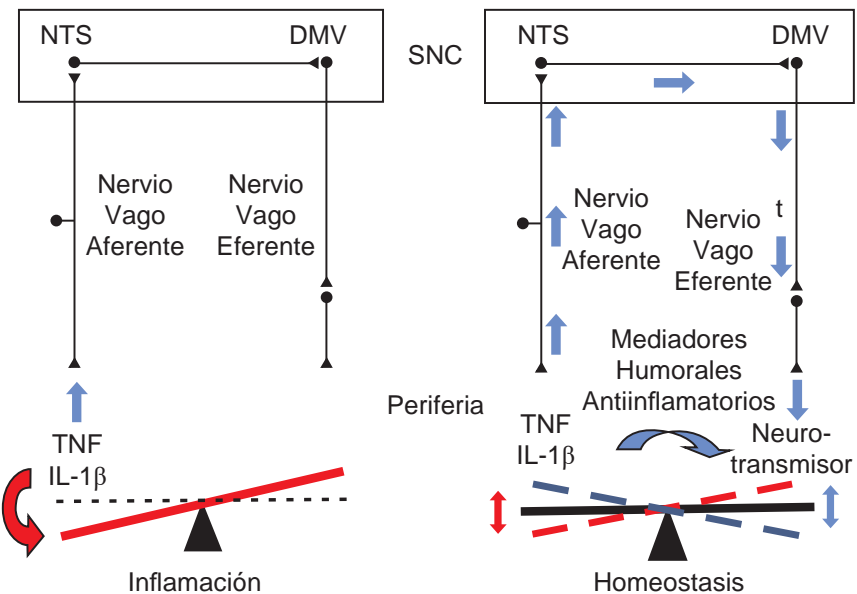

NTS = Núcleo de tracto solitario, SNC = Sistema nervioso central, DMV = Núcleo dorsal motor del vago.

Figura 1: Reflejo inflamatorio: los patógenos y el daño tisular inducen la liberación de citosinas que limitan la extensión de la infección y promueven la reparación tisular. Vías neuronales y humorales regulan la magnitud de la inflamación. La liberación de citosinas en el sitio de inflamación activa las fibras aferentes del nervio vago y alcanza el núcleo tracto solitario en el sistema nervioso central, brindando información proveniente de la periferia. Las señales compensatorias son transportadas por el nervio vago eferente y alcanzan el sitio de la inflamación donde los neurotransmisores actúan sobre los macrófagos y otras células del sistema inmune para atenuar la respuesta inflamatoria.

La actividad de la acetilcolinesterasa puede ser utilizada como biomarcador en el diagnóstico de sepsis debido a infecciones bacterianas, observándose una disminución en pacientes con choque séptico. ${ }^{5}$ Además, se ha evaluado el valor pronóstico de la acetilcolinesterasa junto con la escala APACHE II en pacientes con sepsis, ${ }^{6}$ encontrándose valores similares en predicción de resultados. La utilidad de la actividad de acetilcolinesterasa está directamente relacionada con el aumento de la inflamación secundaria a sepsis, por lo que puede ser una herramienta útil en el diagnóstico y predicción de esta patología. ${ }^{7}$

Objetivo: Estudiar la utilidad de los niveles de la acetilcolinesterasa en el diagnóstico de sepsis y choque séptico.

\section{MATERIAL Y MÉTODOS}

Se realizó un estudio observacional, prospectivo, en la Unidad de Cuidados Intensivos del Hospital San Ángel Inn Universidad. Se realizó consentimiento informado de todos los pacientes ingresados al estudio, o de sus representantes legales.

Para la determinación de la acetilcolinesterasa se utilizó el método fotocolorimétrico, a través del equipo Cobras 6000, utilizando en las muestras sanguíneas EDTA (ácido etilendiaminotetraacético).
Se incluyeron pacientes ingresados a terapia intensiva del Hospital San Ángel Inn Universidad en el periodo comprendido de junio 2016 a junio 2017, con diagnóstico de inestabilidad hemodinámica definida como índice de choque mayor de 1, lactato sérico mayor de $4 \mathrm{mmol} / \mathrm{L}$, empleo de vasopresores después de reanimación con 20 $\mathrm{mL} / \mathrm{kg}$ de peso de cristaloides. Se estableció diagnóstico de choque séptico ${ }^{8}$ conforme la definición de sepsis 3 : presencia de dos o más fallas orgánicas atribuibles a un proceso infeccioso. El diagnóstico de choque hipovolémi$\mathrm{Co}^{9}$ se estableció de acuerdo a los criterios del Colegio Americano de Cirujanos, con la presencia de hipoperfusión tisular secundaria a disminución de la precarga; para este estudio se corroboró choque hipovolémico por los siguientes datos: índice de choque $>1$, déficit de base $<-5$ y presión sistólica $<90 \mathrm{mmHg}$ y colapso de vena cava mayor de $50 \%$. Se estableció choque cardiogénico ${ }^{10}$ conforme los criterios del American College of Cardiology. Para fines de este estudio se corroboró el diagnóstico de cada tipo de choque con la revisión final del caso al egreso hospitalario por parte de dos intensivistas con cinco años de experiencia pertenecientes al Colegio Mexicano de Medicina Crítica y con certificación vigente del Consejo Mexicano de Medicina Crítica.

Se clasificó a los pacientes en tres grupos de estudio: choque séptico, choque hipovolémico y choque cardiogénico. Se excluyeron pacientes que presentaran infecciones fúngicas, virales, envenenamiento con órgano fosforados, cirrosis, tuberculosis activa, trauma craneoencefálico grado II o mayor y muerte cerebral.

\section{Análisis estadístico}

Para el análisis estadístico se dividió a la población en dos grupos: con sepsis (Sp) y sin sepsis (NSp). Se realizó la prueba de Shapiro-Wilk para determinar el tipo de distribución de las variables continuas. Las comparaciones entre grupos se llevaron a cabo mediante prueba $t$ de Student o Mann-Whitney de acuerdo a la distribución normal o no normal, respectivamente.

Para variables cualitativas se realizaron pruebas de $\chi^{2}$ o exacta de Fisher.

Finalmente, se corrieron curvas Recaiver Operating Characteristics de las variables acetilcolinesterasa, linfocitos, neutrófilos e índice neutrófilos/linfocitos contra la presencia de sepsis; se muestran los valores de $p$, áreas bajo la curva, y para aquellas con una diferencia significativa a un área bajo la curva de $50 \%$ se muestran los puntos de corte propuestos con sensibilidad y especificidad.

Las variables cuantitativas con distribución normal se presentan como media (desviación estándar), no normal como mediana (percentil 25- percentil 75) y cuantitativas como frecuencia (porcentaje); se consideró significativa toda $p<0.05$. 


\section{RESULTADOS}

En total se evaluaron $n=44$ pacientes. $S p n=12$ $(27 \%)$ y NSp $32(73 \%)$. Sp $n=6$ masculinos $(50 \%)$ y 6 femeninos (50\%). Nsp predominó el género femenino $\mathrm{n}=24$ pacientes $(75 \%)$. De los 12 pacientes del grupo Sp, $10(83.3 \%)$ no presentaron SIRS, mientras que dos pacientes (16.7\%) sí lo hicieron. Veinticinco por ciento tuvo necesidad de uso de ventilación mecánica invasiva. La escala SOFA fue de 2 puntos (1-5). EI resto de las características se muestra en Tabla 1.

En el grupo Sp, el valor medio de la actividad de la acetilcolinesterasa sérica fue de $3386(+1604)$; la mediana de la proteína $C$ reactiva (PCR) fue de 15.75 (0.1118.70) y la mediana de procalcitonina de 1.93 (1.00-7.87).

El valor de la mediana de la relación $\mathrm{SpO}_{2} / \mathrm{FiO}_{2}$ para el grupo Sp fue de 304.67 (223.75-321.50) y la media de $\mathrm{SpO}_{2}$ fue de $96( \pm 3)$.

Tabla 1: Variables demográficas, bioquímicas, gasométricas y características generales.

\begin{tabular}{|c|c|c|c|}
\hline & \multicolumn{3}{|c|}{ Grupo } \\
\hline & No sepsis (32) & Sí sepsis (12) & Total (44) \\
\hline \multicolumn{4}{|l|}{ Variables demográficas } \\
\hline Edad & $69(64-78)$ & $72(64-77)$ & $70(64-78)$ \\
\hline \multicolumn{4}{|l|}{ Género } \\
\hline Masculino & $8(25)$ & $6(50)$ & $14(31.8)$ \\
\hline Femenino & $24(75)$ & $6(50)$ & $30(68.2)$ \\
\hline \multicolumn{4}{|l|}{ Choque } \\
\hline No & $25(78.1)$ & $8(66.7)$ & $33(75)$ \\
\hline Sí & $7(21.9)$ & $4(33.3)$ & $11(25)$ \\
\hline SOFA & $1(0-2)$ & $2(1-5)$ & $1(0-2)$ \\
\hline qSOFA & $1(0-1)$ & $0(0-1)$ & $1(0-1)$ \\
\hline \multicolumn{4}{|l|}{ SIRS } \\
\hline No & $26(83.9)$ & $10(83.3)$ & $36(83.7)$ \\
\hline Sí & $5(16.1)$ & $2(16.7)$ & $7(16.3)$ \\
\hline \multicolumn{4}{|l|}{ Vasopresores } \\
\hline No & $25(83.9)$ & $9(75)$ & $35(81.4)$ \\
\hline Sí & $5(16.1)$ & $3(25)$ & $8(18.6)$ \\
\hline \multicolumn{4}{|l|}{$\begin{array}{l}\text { Ventilación } \\
\text { mecánica invasiva }\end{array}$} \\
\hline No & $57(87.1)$ & $9(75)$ & $36(83.7)$ \\
\hline Sí & $4(12.9)$ & $3(25)$ & $7(16.3)$ \\
\hline \multicolumn{4}{|l|}{ Variables bioquímicas } \\
\hline Colinesterasa* U/L & $5,786(1,999)$ & $3,386(1,604)$ & $5,100(2196)$ \\
\hline PCR mg/dL & $9.840(2.70-10.65)$ & $15.75(0.11-18.70)$ & $10.60(2.7-14.00)$ \\
\hline Procalcitonina pcg/dL & $0.510(2.70-3.33)$ & $1.930(1.00-7.87)$ & $0.540(0.50-5.55)$ \\
\hline BUN mg/dL & $21.00(15-35)$ & $22.00(17-36)$ & $21.00(15-36)$ \\
\hline Creatinina mg/dL & $1.080(0.64-1.29)$ & $0.990(0.83-1.20)$ & $1.010(0.73-1.29)$ \\
\hline \multicolumn{4}{|l|}{ Variables gasométricas } \\
\hline $\mathrm{pH}$ & 7.390 (7.34-7.43) & 7.400 (7.24-7.42) & $7.390(7.34-7.43)$ \\
\hline $\mathrm{SpO}_{2} \%$ & $94.00(3)$ & $96.00(3)$ & $94.00(3)$ \\
\hline $\mathrm{FiO}_{2} \%$ & $30.00(30-53)$ & $30.00(30-45)$ & $30.00(30-48)$ \\
\hline $\mathrm{SpO}_{2} / \mathrm{FiO}_{2}$ & $\begin{array}{c}313.33 \\
(182.5-326.00)\end{array}$ & $\begin{array}{c}304.67 \\
(223.75-321.50)\end{array}$ & $\begin{array}{c}311.50 \\
(208.50-324.67)\end{array}$ \\
\hline
\end{tabular}

SOFA = Sequential Organ Failure Assessment, qSOFA = Quick Sequential Organ Failure Assessment, SIRS = Síndrome de respuesta inflamatoria sistémica, PCR $=$ Proteína $\mathrm{C}$ reactiva, $\mathrm{BUN}=$ Nitrógeno ureico en sangre, $\mathrm{SpO}_{2}:$ Saturación periférica de oxígeno, $\mathrm{FiO}_{2}=$ Fracción inspirada de oxígeno, ${ }^{*}=p<0.05$ entre grupos.
En el análisis ROC realizado para evaluar el valor diagnóstico de los niveles de acetilcolinesterasa en la predicción de sepsis se observó que los niveles de acetilcolinesterasa tienen una valía diagnóstica (AUC: 0.852 IC 95\%: 0.726-0.977, p < 0.001), cuyo valor de corte se determinó como $\leq 3,956 \mathrm{U} / \mathrm{L}$, sensibilidad como $81 \%$, especificidad como $75 \%$ (Figura 2).

En el análisis ROC para evaluar el porcentaje de neutrófilos como predictor diagnóstico en sepsis se encontró que también tiene importancia, en menor proporción que la acetilcolinesterasa, con significancia estadística (AUC: 0.710 IC 95\%: 0.512-0.907, $p=0.034$ ); y el valor de corte se determinó como $\leq 74 \%$, sensibilidad como $75 \%$, especificidad como $75 \%$ (Figura 3).

\section{DISCUSIÓN}

En nuestro estudio se observa que el punto de corte de los niveles de acetilcolinesterasa para diagnóstico de sepsis es de 3,956 U/L, alcanzando significancia estadística (AUC: 0.852 IC 95\%: 0.726-0.977, p $<0.001$ ), hallazgos similares a los descritos por Koylu, ${ }^{7}$ en los que el punto de corte es de 6,976 U/L, alcanzando una mejor sensibilidad (96.6 versus $81 \%$ ) y especificidad (94.9 versus $75 \%$ ) que la nuestra, asimismo con significancia estadística (AUC: 0.975 IC 95\%: 0.941-1.010, $p<0.001)$. Probablemente, estos resultados se deban a que el tamaño muestral (58 versus 12 casos sépticos y 39 versus 32 casos no sépticos) fue mayor en dicho estudio. Además, exis-

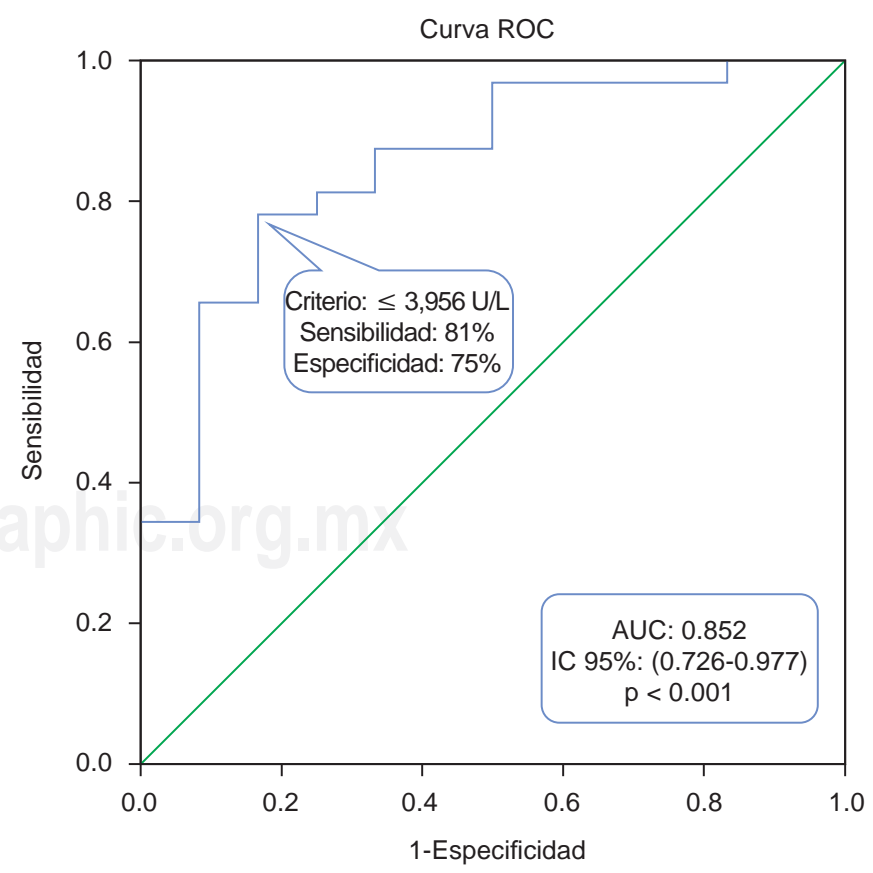

Figura 2: El análisis de la curva ROC de los niveles de colinesterasa como predictor de sepsis. 


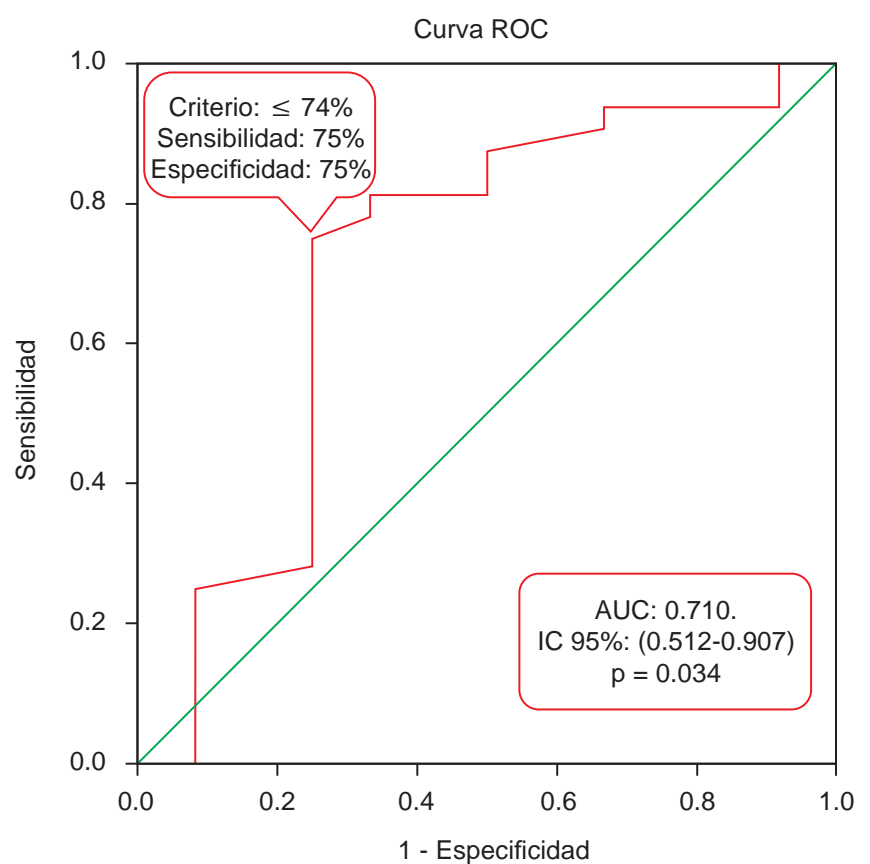

Figura 3: Análisis de la curva ROC del porcentaje de neutrófilos como predictor de sepsis.

ten características de los pacientes sépticos que son equivalentes en ambos estudios: edad ( 71.3 versus 72 ), género masculino (51.7 versus $50 \%$ ), así como el uso de ventilación mecánica invasiva (22.4 versus $25 \%$ ) y uso de vasopresor (41.4 versus $25 \%$ ) en el estudio citado y el nuestro respectivamente. Además, Koylu encontró que la acetilcolinesterasa sirve como marcador pronóstico en la mortalidad, con un corte $\leq 4,018 \mathrm{U} / \mathrm{L}$ con sensibilidad $78.6 \%$ y especificidad 60\% (AUC: 0.699 IC 95\%: 0.563-0.834, $p=0.09$ ). ${ }^{7}$

En un estudio realizado por Feng y colegas, ${ }^{6}$ se identificó que la acetilcolinesterasa era mucho mayor en los pacientes diagnosticados con sepsis que respondieron al tratamiento y sobrevivieron, en comparación con aquellos que fallecieron.

En el estudio de Bahloul ${ }^{5}$ se encontró un menor nivel de acetilcolinesterasa en los pacientes sépticos $(2,923 \pm 931 \mathrm{U} / \mathrm{L}$ versus $3,386 \pm 1,604 \mathrm{U} / \mathrm{L})$; probablemente se deba a que los pacientes incluidos presentaban diferentes características en comparación con los nuestros, ya que aparentemente en este estudio se encontraban más graves, determinados por el valor de SOFA (8.28 \pm 3.5 versus 2 [2-5]), SIRS (100 versus $16.7 \%$ ), uso de vasopresores (61 versus $25 \%$ ) y ventilación mecánica invasiva (72 versus $25 \%$ ). También los niveles de biomarcadores se encontraron muy diferentes, encontrándose mayor elevación de la procalcitonina (25.8 \pm 36 versus 1.93 [1.00-7.87]) y PCR (182 \pm 124 versus 15.75 [0.11-18.70]) en ese estudio. A pesar de dichas diferencias, se encontró que la acetilcolinesterasa fue más efectiva que la procalcitonina y PCR para el diagnóstico de choque séptico, cuando sus valores son $<4,000 \mathrm{U} / \mathrm{L}$, al igual que en nuestro estudio.

Al-Kassab y colaboradores, ${ }^{11}$ expresaron que la reducción de la actividad de la acetilcolinesterasa puede ser un marcador específico de disfunción hepática y síndrome de sepsis sistémica en pacientes ingresados en cuidados intensivos con diagnóstico de choque séptico. También lo indican Chiarla y sus colegas, quienes identificaron una disminución significativa en la actividad de la acetilcolinesterasa de pacientes en estado crítico; en particular, pacientes con sepsis o disfunción hepática. ${ }^{12}$

Hasta donde tenemos conocimiento, nuestro estudio es el primero en reportar la acetilcolinesterasa como biomarcador en el diagnóstico en pacientes sépticos de una población mexicana; sin embargo, presentamos algunas limitaciones que deben ser mencionadas: la naturaleza de un estudio de un solo centro y la limitación de la población.

Nuestro estudio no tiene el poder estadístico para el análisis de mortalidad; sin embargo, con los resultados de otros estudios ${ }^{5-7,12}$ se sugiere que podríamos obtener significancia al ampliar la población y evaluar dicha variable.

\section{CONCLUSIONES}

En presencia de inestabilidad hemodinámica, niveles de acetilcolinesterasa iguales o superiores a 4,000 U/L descartan sepsis como la causa del choque. Son necesarios estudios adicionales con una mayor muestra de pacientes para confirmar nuestros hallazgos, así como su validación externa.

\section{BIBLIOGRAFÍA}

1. Uzzan B, Cohen R, Nicolas P, Cucherat M, Perret GY. Procalcitonin as a diagnostic test for sepsis in critically ill adults and after surgery or trauma: a systematic review and metaanalysis. Crit Care Med. 2006;34(7):1996-2003.

2. Rosas-Ballina M, Tracey KJ Cholinergic control of inflammation. $J$ Intern Med. 2009;265:663-679.

3. Berthoud HR, Neuhuber WL. Functional and chemical anatomy of the afferent vagal system. Auton Neurosci. 2000;85:1-17.

4. Wang $H, Y u M$, Ochani $M$, Amella CA, Tanovic $M$, Susarla S, et al. Nicotinic acetylcholine receptor alpha7 subunit is an essential regulator of inflammation. Nature. 2003;421(6921):384-388.

5. Bahloul M, Baccouch N, Chtara K, Turki M, Turki O, Ben Hamida $\mathrm{C}$, et al. Value of serum cholinesterase activity in the diagnosis of septic shock due to bacterial infections. J Intensive Care Med. 2016;pii:0885066616636549.

6. Feng W, Tang C, Guo H, Bao Y, Wen X, Xue T, et al. Prognostic value of serum cholinesterase activities in sepsis patients. Hepatogastroenterology. 2012;60(125):1001-1005.

7. Oznur Koylu, Mehmet Yortanli. The effect of cholinesterase activity on the diagnosis and prognosis of sepsis. Clinical Medicine Research. 2016;5(3):28-34.

8. Singer M, Deutschman CS, Seymour CW, Shankar-Hari M, Annane D, Bauer M, et al. The third international consensus 
definitions for sepsis and septic shock (Sepsis-3). JAMA. 2016;315(8):801-810.

9. Lawton LD, Roncal S, Leonard E, Stack A, Dinh MM, Byrne CM, et al. The utility of Advanced Trauma Life Support (ATLS) clinical shock grading in assessment of trauma. Emerg Med J. 2014;31(5):384-389.

10. Levy B, Bastien O, Karim B, Cariou A, Chouihed T, Combes $A$, et al. Experts' recommendations for the management of adult patients with cardiogenic shock. Ann Intensive Care. 2015;5(1):52.

11. Al-Kassab A, Vijayakumar E. Profile of serum cholinesterase in systemic sepsis syndrome (septic shock) in intensive care unit patients. Clinical Chemistry and Laboratory Medicine. 1995;33(1):11-14.

12. Chiarla C, Giovannini I, Giuliante F, Vellone M, Ardito F, Nuzzo G. Plasma cholinesterase correlations in acute surgical and critical illness. Minerva Chirurgica. 2011;66(4):323-327.

\section{Patrocinios: No. \\ Relación de conflictos de intereses: No.}

\author{
Correspondencia: \\ Enrique Monares Zepeda \\ Av. Río Churubusco Núm. 601, \\ Col. Xoco, 03339, CDMX. \\ Tel.: 56236363 , ext. 3209 \\ Celular: 0445549946531 \\ E-mail: enrique.monares@hsai.com.mx
}

